\title{
Prevaricare si può!
}

\section{Vittorio Coletti}

PUBBLICATO: 15 MARZO 2019

\begin{abstract}
Quesito:
Prevaricare o prevalicare? È transitivo o intransitivo? E se è intransitivo, con quale preposizione si introduce l'eventuale complemento? E ancora: prevaricatore o prevaricatorio, qual è l'aggettivo giusto?
\end{abstract}

\section{Prevaricare si può!}

Cominciamo dalla domanda sulla forma del verbo: prevaricare e prevalicare sono lo stesso verbo; la seconda è solo una variante antica e popolare (probabilmente attirata da prevalere e/o da travalicare) della prima, che è quella etimologica dal lat. praevaricari, alla lettera 'allargare le gambe (varicari) oltre misura (prae)', da cui il significato figurato poi prevalso di 'andare oltre i (giusti) limiti, eccedere, trasgredire' e, oggi, 'abusare della propria forza o potere ai danni di qualcuno'.

La domanda se il verbo è transitivo o intransitivo è ben fondata: oggi è prevalentemente intransitivo, senza complemento o col complemento indiretto di chi o di ciò che è oggetto della prevaricazione; ma anticamente è stato soprattutto transitivo e principalmente come tale lo registra il Corpus OVI, col significato e il costrutto transitivo di 'trasgredire: "le prime creature per suo (del diavolo) consiglio, il precetto del loro creatore miserabilemente prevaricarono" (Boccaccio, Filocolo).

Oggi è invece quasi solo intransitivo e si pone il problema della reggenza del suo complemento quando esplicitato. In passato erano abbastanza frequenti contro, come si legge in un antico volgarizzamento trecentesco della Bibbia registrato dall'OVI, nel senso di ribellarsi, disobbedire: "coloro i quali hanno prevaricato contro a me", e in: "profanar ... la legge vuol dire contaminarla e in essa prevaricare" (G.B. Guarini nel Cinquecento, dal GDLI), ma anche, più raramente, da, nel significato di "deviare, scostarsi" (come ricorda il Tommaseo-Bellini, da cui si cita): "e qui mi bisogna alquanto prevaricare dal mio assunto"(A.M. Salvini nel 7oo).

Oggi il significato più frequente del verbo è 'abusare, approfittare della propria autorità, prestigio potere, per imporsi su qualcuno o qualcosa' e in questo valore preferisce la preposizione $s u$, forse anche per attrazione di prevalere che la ha adottata. Gli esempi sono quasi tutti recenti e recentissimi, come da I. Cacciavillani, Il Settecento veneziano, 2009: "I suoi interessi corporativi prevaricarono su quelli dello stato". Se ho visto bene l'attestazione più antica offerta da Google Libri risale a un articolo su "Studi economici" del 1957: "nella società alcuni uomini prevaricarono su altri". Oggi, nel significato e nella funzione più corrente del verbo, su pare dunque la preposizione preferibile.

Quanto all'aggettivo, la forma più attestata nei dizionari è quella stessa del sostantivo, prevaricatore, dal latino praevaricatorem, il solo registrato dal GDLI (ovviamente anche e soprattutto come sostantivo), dalle Crusche e da tutti i dizionari correnti consultati. Nessuno di essi invece riporta la forma prevaricatorio, registrata solo da alcuni vocabolari di sinonimi circolanti in rete. Ora, non sono pochi i sostantivi e aggettivi in -tore (dal latino -torem) accanto ai quali si è sviluppato un equivalente deverbale in -torio (dal latino -torium) nella sola funzione di aggettivo, con lo stesso significato (inibitore / inibitorio, inquisitore / inquisitorio, pacificatore / pacificatorio) oppure con significato leggermente diverso (liberatore / liberatorio, [giudice] istruttore /[procedimento] istruttorio). Quando è disponibile la coppia, la forma in -tore si riserva il ruolo originario (spesso già latino) di sostantivo (preparatore, creditore) e quella in -torio, già latina o, più spesso, ricavata da materiale italiano, di aggettivo (preparatorio, creditorio). La lingua sta provvedendo anche per prevaricatore a questo non disutile sdoppiamento di ruoli e ha propiziato la nascita di prevaricatorio, aggettivo ormai discretamente attestato (comportamento, atto prevaricatorio), nonostante i dizionari non se ne siano ancora accorti. Anch'esso, come la reggenza su del verbo di partenza, è molto recente (su Google Libri trovo uno "strumento prevaricatorio" in "Paragone" 1970), segno di un processo di risistemazione in un ambito semantico, in cui le prevaricazioni, vere o presunte, non mancano, purtroppo. Per questo prevaricatorio si può usare senza problemi. 


\section{Cita come:}

Vittorio Coletti, Prevaricare si può!, "Italiano digitale", 2019, VIII, 2019/1 (gennaio-marzo) , pp. 4445.

DOI: $10.35948 / 2532-9006 / 2019.3077$

Copyright 2019 Accademia della Crusca

Pubblicato con licenza creative commons CC BY-NC-ND 